\title{
Therapy of newly diagnosed follicular lymphoma
}

\section{Jason R. Westin and Sattva S. Neelapu*}

University of Texas MD Anderson Cancer Center, Houston, TX, USA

\section{Edited by:}

Gautam Borthakur, University of Texas MD Anderson Cancer Center, USA

\section{Reviewed by:}

Pierluigi Porcu, The Ohio State

University, USA

Marta Coscia, University of Torino, Italy

\section{*Correspondence.}

Sattva S. Neelapu, University of Texas MD Anderson Cancer Center, 1515

Holcombe Boulevard, Unit 0903,

Houston, TX 77030, USA.

e-mail: sneelapu@mdanderson.org
Newly diagnosed follicular lymphoma is relatively common and can be effectively treated with several differing approaches. Although the disease is often considered incurable, it is highly responsive to therapy when indicated. This review discusses the indications for treatment, risk stratification systems, treatment options with supporting clinical trial data, and expected therapeutic outcomes in newly diagnosed follicular lymphoma.

Keywords: newly diagnosed, B cell, lymphoma, follicular lymphoma, observation, immunotherapy, chemotherapy, outcomes

\section{INTRODUCTION}

Follicular lymphoma is the second most common subtype of lymphoma with nearly 15,000 new cases annually in the United States (Siegel et al., 2011). For unclear reasons, the incidence of FL continues to increase in the United States and Europe (Cartwright et al., 1999; Groves et al., 2000). FL arises from germinal-center B cells with the genetic hallmark of an acquired $t(14 ; 18)(\mathrm{q} 32 ; \mathrm{q} 21)$ translocation, leading to deregulation of $\mathrm{BCL} 2$, a key gene in the regulation of apoptosis and cell death. The $\mathrm{t}(14 ; 18)$ translocation is found in the normal B cells of over half of healthy individuals, and thus is insufficient alone for lymphomagenesis (Staudt, 2007). Occasionally, an in situ type of FL is identified with $\mathrm{t}(14 ; 18)$ and BCL2 expression in a lymph node without other sites of disease. Patients with in situ FL rarely progress to disseminated FL and generally can be observed (Limpens et al., 1991; Cong et al., 2002; Roulland et al., 2006; Staudt, 2007; Jaffe, 2009). Historically, cells harboring the $\mathrm{t}(14 ; 18)$ translocation in the peripheral blood of healthy individuals were thought to represent naïve $\mathrm{B}$ cells, but recent work suggests these cells have many similarities to FL cells, including class-switch recombination, and surface expression of IgM and $\mathrm{IgD}$ (Roulland et al., 2006). It is not clear if the presence of the $t(14 ; 18)$ translocation in healthy individuals has any prognostic or therapeutic value, but suggests a possible common pre-malignant stage.

\section{PROGNOSTIC SYSTEMS}

Nearly five of every six patients with newly diagnosed FL will have advanced disease, either due to a long asymptomatic phase prior to diagnosis or early dissemination. Follicular lymphoma is staged by the Ann Arbor system, originally devised in the 1970s to account for radiation field size in Hodgkin lymphoma. The system accounts for a stepwise progression to adjacent lymph node chains, a pattern often not found in non-Hodgkin lymphoma (NHL), and thus its validity in this population has been questioned (Staudt and Dave, 2005). Clinical outcomes vary within stages, suggesting that clinical factors other than location of disease affect outcome.

In 2000, the Intergruppo Italiano Linfomi attempted to account for these additional factors by proposing a model incorporating age, sex, number of extranodal sites of disease, B symptoms, serum lactate dehydrogenase (LDH), and erythrocyte sedimentation rate (ESR; Federico et al., 2000). This model was further improved by an international cooperative effort evaluating over 4000 patients, creating the Follicular Lymphoma International Prognostic Index (FLIPI, Figure 1). Based on the number of adverse features present, patients were classified into three groups with 10-year overall survival (OS) rates of 70.7, 50.9, and 35.5\% (Solal-Céligny et al., 2004). The FLIPI was widely adopted, but had several important limitations. It was created from a retrospective database analysis of patients not treated with current chemoimmunotherapy, was missing significant amounts of data (ESR, $\beta 2 \mathrm{M}$, and performance status), and had an endpoint of OS. OS is difficult to model in an indolent disease such as FL due to the duration of follow-up required, a relapsing and remitting disease course, and multiple lines of effective therapy. To account for these weaknesses, the International Follicular Lymphoma Prognostic Factor Project launched the F2 study in 2003 (Federico et al., 2009). The primary endpoint of the model was progression-free survival (PFS), now the preferred metric in lymphoma clinical trials (Cheson et al., 2007). The factors identified as significant in the F2 study, now referred to as the FLIPI2, are relatively similar to those included in the FLIPI score (Figure 1). Based on the number of factors present, the FLIPI2 classifies patients into three groups with 5-year PFS of $79.5,51.2$, and $18.8 \%$.

It is important to note that the vast majority of patient data utilized to generate these models is from patients with grades 1 and 2 disease. Grade $3 \mathrm{FL}$ is split into A and B categories by the WHO classification. The B category lacks centrocytes and has similar biology and outcomes to diffuse large B cell lymphoma (DLBCL; Jaffe, 2009). Grade 3B FL is generally treated like DLBCL, however this remains an area of controversy. The discussion in this paper refers to $\mathrm{FL}$ grades $1-3 \mathrm{~A}$, unless otherwise stated.

As the majority of patients have a responding and relapsing course, the option of not treating immediately after diagnosis, often referred to as "watch and wait," was explored and found to have no detrimental effect on OS (Horning and Rosenberg, 1984; 


\section{GELF Criteria}

Any Node $>7 \mathrm{~cm}$
3 or more nodes $>3 \mathrm{~cm}$ each
B symptoms
Splenomegaly
Leukocytosis or Leukemic phase
Pleural or peritoneal effusions

FLIPI

Age $>60$
Hemoglobin $<12 \mathrm{~g} / \mathrm{L}$
Elevated LDH
Nodal sites $>4$
Stage III/IV

\section{FLIPI2}

Age $>60$
Hemoglobin $<12 \mathrm{~g} / \mathrm{L}$
Elevated $\beta 2 \mathrm{M}$
Any node $>6 \mathrm{~cm}$
Bone Marrow +

FIGURE 1 | Follicular lymphoma therapy indications and clinical predictors.

O'Brien et al., 1991). More concerning, early retrospective reviews found that multiple single agents and chemotherapy combinations, though initially effective, had little or no effect on the OS of patients with FL (Horning, 1993). Newer studies which include therapy with targeted agents have demonstrated improvements in survival; however cure remains elusive for the vast majority of FL patients. Thus, newer treatments which prolong remission duration with minimal toxicity are needed.

\section{INDICATIONS FOR THERAPY}

Most cases of FL demonstrate an indolent nature, and when treated are highly responsive to therapy (Cheson, 2010). Nearly all advanced FL will eventually relapse after response to initial therapy, and thus FL is currently considered incurable in most cases.

After appropriately staging a newly diagnosed patient with FL, the most important initial decision is "Does this patient require therapy now?" To assist in answering this question, several risk stratification classification systems have been generated for FL. The Groupe d'Etude des Lymphomes Folliculaires (GELF) criteria (Figure 1) were developed in a prospective clinical trial randomizing patients to observation, chemotherapy, or interferon (Brice et al., 1997). In this trial, patients with a high tumor burden had a significantly worse OS (5-year: 78 vs. 57\%). Based on these data, patients with $\geq 3$ nodal sites with diameters of $\geq 3 \mathrm{~cm}$ or one mass $\geq 7 \mathrm{~cm}$ are typically not candidates for delayed therapy.
If a patient does not require immediate treatment based on the above criteria, the options include "watch and wait," immunotherapy with or without chemotherapy, and or radiotherapy.

\section{WATCH AND WAIT}

Deferred therapy with serial observation, or "watch and wait," has been employed in asymptomatic patients with low disease burden due to the typically indolent nature of FL, occasionally even demonstrating regression or long periods of disease stability without therapy (Horning and Rosenberg, 1984). Spontaneous regressions, occurring in 5-25\% patients, are thought to be related to the immune responsive nature of FL (Kannan and Neelapu, 2009). In the GELF-86 trial, patients with newly diagnosed FL were randomized to receive interferon, the oral alkylating agent prednimustine, or observation until clinically meaningful progression (Brice et al., 1997). The overall response rate (ORR) to therapy was 70 and $78 \%$ in the early treatment arms, and $70 \%$ once therapy was indicated in the observation arm, and OS at 5 years was similar in all three arms. In another immediate vs. delayed therapy clinical trial, nearly $10 \%$ of the patients randomized to the observation arm did not require systemic therapy with at least 10 years of follow-up (Ardeshna et al., 2003). Several other trials have demonstrated similar outcomes with initial and delayed therapy (Abramson et al., 2009; Kloo et al., 2011). Even with modern therapies yielding improved survival, there are no conclusive data yet which suggest "watch and wait" results in inferior long-term survival outcomes.

The risk of transformation to a more aggressive form of lymphoma is a dreaded event in FL patients, as even intensification of therapy typically results in poor outcomes with the majority of patients dying within 2 years (Yuen et al., 1995). A large retrospective observational study found the annual risk of transformation to be an estimated 3\% per year in patients initially treated with chemotherapy, radiotherapy, or observation (Al-Tourah et al., 2008).

An important factor to consider with the "watch and wait" strategy is patient anxiety. It is certainly understandable that some patients may find it difficult to receive a diagnosis of cancer and be given a follow-up visit in lieu of cancer therapy. Patient anxiety is often lessened when hearing the rationale behind "watch and wait," and the advantages of deferring exposure to potential treatmentassociated toxicities.

\section{RADIOTHERAPY}

Limited stage FL (stage I and contiguous stage II) is potentially curable with radiotherapy alone. Several series have reported dramatic long-term disease-free survival (35-50\% at $\geq 10$ years, with $100 \%$ local control in the radiation field), likely representing cure, in patients who were treated with involved field radiotherapy with minimal long-term toxicities (Mac Manus and Hoppe, 1996; Wilder etal., 2001). A large trial of aggressive chemotherapy (prior to rituximab) with 30-40 Gy involved field radiotherapy resulted in a 10 -year time to treatment failure and OS rates of 72 and $80 \%$, respectively (Seymour et al., 2003). A randomized trial is currently evaluating radiotherapy with and without chemotherapy in this population. As neither chemotherapy nor immunotherapy are currently able to achieve this degree 
of long-term disease control, radiotherapy is considered the standard of care and should be strongly considered in this patient population.

\section{IMMUNOTHERAPY}

The immune responsive nature of certain malignancies, including FL, has been successfully manipulated for therapeutic benefit using multiple strategies. In the late ninetieth century, Dr. William Coley used a mixture of bacterial toxins as an initial attempt at immunotherapy and reportedly achieved dramatic results in lymphomas. Unfortunately, these results could not be replicated (Thotathil and Jameson, 2007). The immune stimulant interferon$\alpha$ demonstrated significant activity in follicular lymphoma, either in combination with chemotherapy or alone, but did not have a clear impact on OS and is not commonly utilized due to treatment related toxicities (Price et al., 1991; Solal-Celigny et al., 1993; Solal-Céligny et al., 1998; Herold et al., 2007; Baldo et al., 2010).

Dr. Levy and others proposed using monoclonal antibodies, a passive immunization strategy, to target lymphoma cell surface markers. In 1994, the phase I trial of a monoclonal antibody targeting the B cell marker CD20 (IDEC-C2B8, rituximab) displayed tumor regression in $40 \%$ of heavily pre-treated B cell lymphoma patients (Maloney et al., 1994). The mechanism of action of rituximab is thought to be a combination of complement-mediated cytotoxicity, antibody-dependent cellular cytotoxicity, and direct signaling. Rituximab has shown significant single agent activity in both the frontline and relapse settings (Table 1; Maloney et al., 1997; McLaughlin et al., 1998; Witzig et al., 2005). Importantly, these multicenter trials also found rituximab to be well tolerated with major toxicities essentially limited to infusional reactions.

In an attempt to directly address "watch and wait" vs. immediate immunotherapy, an intergroup trial randomized newly diagnosed FL patients with stage $\geq$ II, asymptomatic non-bulky disease to observation, weekly rituximab $\times 4$ doses, or weekly rituximab $\times 4$ doses followed by maintenance rituximab (MR) every 2 months for 2 years (Ardeshna et al., 2010). The primary endpoints were time to initiation of new therapy and quality of life. Preliminary results show the estimated median time to initiation of new therapy was 33 months in the observation arm and not reached at 4 years in the rituximab arms. Perhaps not surprisingly, no difference in OS was found between the groups. It remains unclear if the delay in time to initiation of new therapy achieved by

Table 1 | Clinical outcome with various therapeutic regimens in newly diagnosed FL (top) and promising experimental therapies in development in FL (bottom).

\begin{tabular}{|c|c|c|c|c|}
\hline & ORR & CR & PFS (month) & Reference \\
\hline Previously treated & $46-48$ & $6-8$ & $10-13$ & Maloney etal. (1997), \\
\hline Untreated & 72 & 36 & 26 & Witzig et al. (2005) \\
\hline $\mathrm{R}-\mathrm{CHOP}$ & 96 & 20 & NR, 30 months $=75 \%$ & Hiddemann et al. (2005) \\
\hline$B R$ & 92.7 & 39.8 & 69.5 & Rummel et al. (2012) \\
\hline $\mathrm{R}-\mathrm{CHOP}$ & 91.3 & 30 & 31.2 & Rummel et al. (2012) \\
\hline $\mathrm{R}-\mathrm{CHOP}$ & 85 & 41 & $N R, 2$ years $=76 \%$ & Press et al. (2011) \\
\hline CHOP-RIT & 83 & 46 & $N R, 2$ years $=80 \%$ & Press et al. (2011) \\
\hline CAL 101 & P13K & Phase II & & de Vos etal. (2011) \\
\hline ABT-263 & $\mathrm{Bcl} 2$ & Phase I/II & & Wilson et al. (2009) \\
\hline Epratuzumab & CD22 & Phase II & & Leonard et al. (2008) \\
\hline Inotuzumab & CD22 & Phase III & & Ogura etal. (2010) \\
\hline SAR3419 & CD19 & Phase I & & Younes et al. (2009) \\
\hline SGN-40 & $\mathrm{CD} 40$ & Phase I & & Advani etal. (2006) \\
\hline Blinatumomab & CD19/T cell & Phase I & & Viardot et al. (2010) \\
\hline Pidilizumab & PD-1 & Phase II & & Westin et al. (2010) \\
\hline PF-05082566 & $4-1 B B$ & Phase I & & Fisher et al. (2012) \\
\hline
\end{tabular}

ORR, overall response rate; $C R$, complete response; PFS, progression free survival; $R I T$, radioimmunotherapy. 
initial rituximab will ultimately change the natural history of the disease, future responses to rituximab, or time to second line therapy.

The PRIMA study demonstrated the value of continued exposure to rituximab in a less frequent schedule, commonly referred to as maintenance therapy (Salles et al., 2011). Over a thousand patients with newly diagnosed FL who achieved CR or PR after chemoimmunotherapy were randomized to observation or rituximab once every 2 months. At 2 years, $75 \%$ of patients treated with rituximab maintenance were free from progression, as opposed to $58 \%$ of observation patients $(p<0.0001)$. The RESORT trial randomized newly diagnosed FL patients who responded to rituximab weekly $\times 4$ to receive indefinite MR or rituximab retreatment (RR) at the time of disease progression (Kahl et al., 2011). The median time to treatment failure was 3.9 years with MR and 3.6 with $\mathrm{RR}$, and $95 \%$ of $\mathrm{MR}$ and $86 \%$ of $\mathrm{RR}$ did not require cytotoxic chemotherapy at 3 years. The mean number of rituximab doses were quite different: the MR group received 15.8 (range 5-31) and RR 4.5 (range 4-16) doses, raising the question of the cost effectiveness of the maintenance approach. No difference in OS was observed in either the PRIMA or RESORT trials.

Lenalidomide, an immunomodulatory agent that is FDA approved to treat multiple myeloma, has recently shown very promising efficacy in both newly diagnosed and relapsed FL (Witzig et al., 2009; Fowler et al., 2010; Zhu et al., 2011; Leonard et al., 2012). A phase II study of lenalidomide with rituximab in newly diagnosed FL patients revealed an impressive preliminary ORR of $98 \%$ and CR of $87 \%$ (Fowler et al., 2010, 2011). An international randomized trial comparing rituximab with either lenalidomide or chemotherapy in newly diagnosed FL is currently underway.

\section{CHEMOTHERAPY}

Follicular lymphoma is a chemosensitive disease, with numerous combinations successfully utilized over the past 30 years. A retrospective review spanning 25 years from our institution found that with each subsequent intensification of initial therapy, both failure-free survival (FFS) and OS improved (Liu et al., 2006). The 1972-1982 cohort was treated with cyclophosphamide, doxorubicin, vincristine, prednisone (CHOP), and bleomycin, which resulted in a 15-year FFS and OS of 13 and 27\%, respectively. In contrast, the 1988-1992 cohort was treated with intensive alternating triple therapy and interferon, and achieved a 15-year FFS and OS of 32 and 50\%, respectively. Newer therapies, including those with rituximab, had not yet reached their median FFS and OS at the time of publication but appeared to result in further improvements. As with any study measuring long-term outcomes over different eras, a caveat is required for the possible influence of improved supportive care and subsequent therapies on survival.

The addition of rituximab to less intensive chemotherapy regimens, including CHOP and CVP, has proven in multiple studies to be superior to chemotherapy alone (Czuczman et al., 2004; Marcus et al., 2008). A randomized trial by the German Low-Grade Lymphoma Study Group of CHOP with or without rituximab found superior response rates, duration of response, and OS in the rituximab arm (Table 1; Hiddemann et al., 2005). As a result, rituximab is now established as a core component of nearly every therapy for FL, both as a single agent and combined with chemotherapy.

The combination of rituximab and bendamustine, an unique drug with activity similar to alkylating agents and purine analogs, has demonstrated significant activity in untreated and relapsed FL (Table 1; Rummel et al., 2005; Cheson and Rummel, 2009). A randomized study of rituximab combined with bendamustine or CHOP in newly diagnosed FL reported a superior PFS of 69.5 vs. 31.2 months favoring bendamustine (Rummel et al., 2012). Although the final report of this trial has not been published to date, the combination of bendamustine and rituximab is now commonly used for newly diagnosed patients requiring therapy.

\section{RADIOIMMUNOTHERAPY}

In patients with newly diagnosed advanced stage FL treated with CHOP chemotherapy (without rituximab), a single dose of ${ }^{131} \mathrm{I}$ tositumomab resulted in an ORR of $91 \%$ and CR of $69 \%$ (Press et al., 2006). A randomized phase III study of R-CHOP vs. CHOP followed by ${ }^{131}$ I-tositumomab achieved similar results (2-year PFS of 76 and 80\%, OS 97 and 93\%, respectively; Press et al., 2011). An ongoing trial of R-CHOP followed by radioimmunotherapy consolidation and MR aims to determine if efficacy can be further improved by combining multiple anti-CD20 therapeutic modalities. A single course of ${ }^{131}$ I-tositumomab in newly diagnosed FL without chemotherapy resulted in an ORR of 95\%, CR of $75 \%$, and a median PFS of 6.1 years, an approach which may be valuable to elderly patients who fail rituximab monotherapy and decline chemotherapy (Kaminski et al., 2005).

\section{POST-THERAPY FOLLOW-UP}

According to the NCCN guidelines, patients with initially treated FL should be followed with physical exam and laboratory assessment every 3-6 months for at least 5 years. Surveillance CT scans should be performed no more than every 6 months for the first 2 years, then annually if indicated. Patients and physicians may feel more comfortable with frequent imaging, but the associated cost and radiation exposure are not likely more advantageous than early diagnosis of relapse in this indolent disease. When relapse is identified, immediate therapy is not required and the same general criteria used in newly diagnosed patients can aide in determining when to start therapy.

If a patient appears to have relapsing disease with a rapidly enlarging site, significantly elevated LDH, or new B symptoms, it is possible they have transformed into an aggressive lymphoma (Wong and Dickinson, 2012). In this setting, it is imperative to obtain a biopsy of the concerning site, as the most accessible lymph node may still represent low-grade FL. Patients with FL which has undergone transformation have a poor prognosis and should be considered for a clinical trial.

\section{OUTCOMES}

The National LymphoCare Study evaluated treatment practices of academic and community oncologists and found that $17 \%$ opted for initial observation, $13.9 \%$ rituximab monotherapy, $51.9 \%$ rituximab with chemotherapy, 5.6\% radiation therapy, and only $6.1 \%$ on a clinical trial. These data were obtained prior 
to the data regarding bendamustine, and thus the majority of chemotherapy-treated patients received R-CHOP. With the perception that bendamustine has less toxicity than CHOP, these data have likely shifted to include more use of chemotherapy overall, with CHOP making up a smaller proportion. Of note, only $23 \%$ of stage I patients received radiation therapy - a potentially curative approach (see Radiation).

Newly diagnosed FL patients often are confused regarding their long-term prognosis, as FL is often considered “incurable." It is important to clarify the important difference between incurable and untreatable. Patients may read information on the Internet and not understand this difference, and thus be unnecessarily concerned or question the recommendations of their doctor. The median OS for all newly diagnosed FL patients is $>10$ years, and these data are not likely representative of modern therapy as they are based on patients diagnosed in the pre-rituximab era (Liu et al., 2006).

\section{INVESTIGATIONAL APPROACHES}

The LymphoCare study found that only $6.1 \%$ of patients were enrolled on a clinical trial for newly diagnosed FL. This may be due in part to the perception that FL is easily treated with standard therapies that achieve satisfactory outcomes. Although we agree with these assumptions, we recommend all newly diagnosed patients with FL be considered for clinical trial enrollment.

\section{REFERENCES}

Abramson, J. S., Chen, W., Juszczynski, P., Takahashi, H., Neuberg, D. Kutok, J. L., et al. (2009). The heat shock protein 90 inhibitor IPI-504 induces apoptosis of AKT-dependent diffuse large B-cell lymphomas. Br. J. Haematol. 144, 358-366.

Advani, R., Forero-Torres, A., Furman, R. R., Rosenblatt, J. D., Younes, A., Shankles, B., et al. (2006). SGN-40 (anti-huCD40 mAb) monotherapy induces durable objective responses in patients with relapsed aggressive non-Hodgkin's lymphoma: evidence of antitumor activity from a phase I study. Blood 108, abstr. 695.

Advani, R. H., Buggy, J. J., Sharman, J. P., Smith, S. M., Boyd, T. E., Grant, B., et al. (2012). Bruton tyrosine kinase inhibitor ibrutinib (PCI-32765) has significant activity in patients with relapsed/refractory B-cell malignancies. J. Clin. Oncol. doi: 10.1200/jco.2012.42.7906 [Epub ahead of print].

Al-Tourah, A. J., Gill, K. K., Chhanabhai, M., Hoskins, P. J., Klasa, R. J., Savage, K. J., et al. (2008). Population-based analysis of incidence and outcome of transformed non-Hodgkin's lymphoma. J. Clin. Oncol. 26, 51655169.

Ardeshna, K., Smith, P., Qian, W., Warden, J., Stevens, L., Pocock, C., et al. (2010). An intergroup randomised trial of rituximab versus a watch and wait strategy in patients with stage II, III, IV, asymptomatic, non-bulky follicular lymphoma (grades 1, 2 and 3a). A preliminary analysis. Blood 116, abstr. 6

Ardeshna, K. M., Smith, P., Norton, A., Hancock, B. W., Hoskin, P. J., MacLennan, K. A., et al. (2003). Long-term effect of a watch and wait policy versus immediate systemic treatment for asymptomatic advanced-stage non-Hodgkin lymphoma: a randomised controlled trial. Lancet 362, 516-522.

Baldo, P., Rupolo, M., Compagnoni, A. Lazzarini, R., Bearz, A., Cannizzaro, R., etal. (2010). Interferon-alpha for maintenance of follicular lymphoma. Cochrane Database Syst. Rev. CD004629.

Brice, P., Bastion, Y., Lepage, E., Brousse, N., Haioun, C., Moreau, P., et al. (1997). Comparison in lowtumor-burden follicular lymphomas between an initial no-treatment policy, prednimustine, or interferon alfa: a randomized study from the Groupe d'Etude des Lymphomes Folliculaires. Groupe d'Etude des Lymphomes de l'Adulte. J. Clin. Oncol. 15, 1110-1117.

Cartwright, R., Brincker, H., Carli, P. M., Clayden, D., Coebergh, J. W., Jack, A., et al. (1999). The rise in incidence

Follicular lymphoma remains an incurable disease that requires costly, and occasionally toxic, recurrent treatments. An impressive assortment of new targeted agents and immunotherapies are undergoing clinical evaluation (Table 1; Advani et al., 2006, 2012; Leonard et al., 2008; Wilson et al., 2009; Younes et al., 2009; Ogura et al., 2010; Viardot et al., 2010; Westin et al., 2010; de Vos et al., 2011; Schuster et al., 2011; Fisher et al., 2012). The ultimate determination if these potential advances can improve outcomes for patients with FL depends upon having a significant number of both newly diagnosed and relapsed patients enroll on clinical trials.

\section{CONCLUSION}

Follicular lymphoma is a common disease due to its incidence and relatively long life expectancy. Despite it being currently considered incurable, many effective treatments exist with many more under evaluation. Patients with early stage disease should be treated with radiotherapy, which is potentially curative. Newly diagnosed advanced stage disease patients should not reflexively be treated unless they are symptomatic or meet standardized criteria. When treatment is necessary, immunotherapy or immunochemotherapy are very effective, but eventual relapse is common. We recommend all newly diagnosed FL patients be considered for a clinical trial to accelerate the development of the next generation of therapy.

of lymphomas in Europe 1985-1992. Eur. J. Cancer 35, 627-633.

Cheson, B. (2010). Targeted treatment and new agents in follicular lymphoma. Int. J. Hematol. 92, 5-11.

Cheson, B. D., Pfistner, B., Juweid, M. E., Gascoyne, R. D., Specht, L., Horning, S. J., et al. (2007). Revised response criteria for malignant lymphoma. $J$. Clin. Oncol. 25, 579-586.

Cheson, B. D., and Rummel, M. J. (2009). Bendamustine: rebirth of an old drug. J. Clin. Oncol. 27, 14921501.

Cong, P., Raffeld, M., Teruya-Feldstein, J., Sorbara, L., Pittaluga, S., and Jaffe, E. S. (2002). In situ localization of follicular lymphoma: description and analysis by laser capture microdissection. Blood 99, 3376-3382.

Czuczman, M. S., Weaver, R., Alkuzweny, B., Berlfein, J., and GrilloLópez, A. J. (2004). Prolonged clinical and molecular remission in patients with low-grade or follicular nonHodgkin's lymphoma treated with rituximab plus CHOP chemotherapy: 9-year follow-up. J. Clin. Oncol. 22, 4659-4664.

de Vos, S., Schreeder, M. T., Flinn, I. W., Coutre, S. E., Leonard, J. P., WagnerJohnston, N. D., et al. (2011). A phase 1 study of the selective phosphatidylinositol 3-kinase-delta (PI3K $\{$ delta $\}$ ) inhibitor, Cal-101 (GS-1101), in combination with rituximab and/or bendamustine in patients with previously treated, indolent non-Hodgkin lymphoma (iNHL). Blood 118, abstr. 2699.

Federico, M., Bellei, M., Marcheselli, L., Luminari, S., Lopez-Guillermo, A., Vitolo, U., et al. (2009). Follicular lymphoma international prognostic index 2: a new prognostic index for follicular lymphoma developed by the international follicular lymphoma prognostic factor project. J. Clin. Oncol. 27, 45554562.

Federico, M., Vitolo, U., Zinzani, P. L., Chisesi, T., Clò, V., Bellesi, G., et al. (2000). Prognosis of follicular lymphoma: a predictive model based on a retrospective analysis of 987 cases. Blood 95, 783-789.

Fisher, T., Kamperschroer, C., Oliphant, T., Love, V., Lira, P., Doyonnas, R., et al. (2012). Targeting of 4-1BB by monoclonal antibody PF-05082566 enhances T-cell function and promotes anti-tumor activity. Cancer Immunol. Immunother. 61, 17211733.

Fowler, N., Hagemeister, F. B., Mclaughlin, P., Kwak, L., Romaguera, J., Fanale, M., et al. (2011). Lenalidomide plus rituximab is a highly effective and well-tolerated biologic therapy in untreated indolent B-cell non-Hodgkin lymphoma. 
Ann. Oncol. 22(Suppl. 4), iv128iv129.

Fowler, N. H., McLaughlin, P., Hagemeister, F. B., Kwak, L. W., Fanale, M. A., Neelapu, S. S., et al. (2010). Complete response rates with lenalidomide plus rituximab for untreated indolent B-cell non-Hodgkin's lymphoma. J. Clin. Oncol. 28(Suppl. 15), abstr 8036

Groves, F. D., Linet, M. S., Travis, L. B., and Devesa, S. S. (2000). Cancer surveillance series: non-Hodgkin's lymphoma incidence by histologic subtype in the United States from 1978 through 1995. J. Natl. Cancer Inst. 92, 1240-1251.

Herold, M., Haas, A., Srock, S., Neser, S., Al-Ali, K. H., Neubauer, A., et al. (2007). Rituximab added to firstline mitoxantrone, chlorambucil, and prednisolone chemotherapy followed by interferon maintenance prolongs survival in patients with advanced follicular lymphoma: an East German study group hematology and oncology study. J. Clin. Oncol. 25, 1986-1992.

Hiddemann, W., Kneba, M., Dreyling, M., Schmitz, N., Lengfelder, E. Schmits, R., et al. (2005). Frontline therapy with rituximab added to the combination of cyclophosphamide, doxorubicin, vincristine, and prednisone (CHOP) significantly improves the outcome for patients with advanced-stage follicular lymphoma compared with therapy with CHOP alone: results of a prospective randomized study of the German Low-Grade Lymphoma Study Group. Blood 106, 3725-3732.

Horning, S. J. (1993). Natural history of and therapy for the indolent nonHodgkin's lymphomas. Semin. Oncol. 20(Suppl. 5), 75-88.

Horning, S. J., and Rosenberg, S. A. (1984). The natural history of initially untreated low-grade nonHodgkin's lymphomas. N. Engl. J. Med. 311, 1471-1475.

Jaffe, E. S. (2009). The 2008 WHO classification of lymphomas: implications for clinical practice and translational research. Hematology 2009, 523-31.

Kahl, B. S., Hong, F., Williams, M. E., Gascoyne, R. D., Wagner, L. I., Krauss, J. C., et al. (2011). Results of Eastern Cooperative Oncology Group Protocol E4402 (RESORT): a randomized phase III study comparing two different rituximab dosing strategies for low tumor burden follicular lymphoma. Blood 118, abstr. 6 .

Kaminski, M. S., Tuck, M., Estes, J., Kolstad, A., Ross, C. W., Zasadny, K., et al. (2005). 131I-tositumomab therapy as initial treatment for follicular lymphoma. N. Engl. J. Med. 352, 441-449.

Kannan, S., and Neelapu, S. (2009). Vaccination strategies in follicular lymphoma. Curr. Hematol. Malig. Rep. 4, 189-195.

Kloo, B., Nagel, D., Pfeifer, M., Grau, M., Düwel, M., Vincendeau, M., et al. (2011). Critical role of PI3K signaling for NF- $\kappa \mathrm{B}$-dependent survival in a subset of activated B-cell-like diffuse large B-cell lymphoma cells. Proc. Natl. Acad. Sci. U.S.A. 108, 272-277.

Leonard, J., Jung, S., Johnson, J., Bartlett, N., Blum, K., and Cheson, B. (2012). CALGB 50401: randomized trial of lenalidomide alone versus lenalidomide plus rituximab in patients with recurrent follicular lymphoma. J. Clin. Oncol. 30(Suppl.), abstr 8000.

Leonard, J. P., Schuster, S. J., Emmanouilides, C., Couture, F., Teoh, N., Wegener, W. A., et al. (2008). Durable complete responses from therapy with combined epratuzumab and rituximab. Cancer 113, 2714-2723.

Limpens, J., de Jong, D., van Krieken, J. H., Price, C. G., Young, B. D., van Ommen, G. J., et al. (1991). Bcl-2/JH rearrangements in benign lymphoid tissues with follicular hyperplasia. Oncogene 6, 2271-2276.

Liu, Q., Fayad, L., Cabanillas, F., Hagemeister, F. B., Ayers, G. D., Hess, M., et al. (2006). Improvement of overall and failure-free survival in stage IV follicular lymphoma: 25 years of treatment experience at the University of Texas M.D. Anderson Cancer Center. J. Clin. Oncol. 24, 1582-1589.

Mac Manus, M. P., and Hoppe, R. T. (1996). Is radiotherapy curative for stage I and II low-grade follicular lymphoma? Results of a long-term follow-up study of patients treated at Stanford University. J. Clin. Oncol. 14, 1282-1290.

Maloney, D. G., Grillo-López, A. J., White, C. A., Bodkin, D., Schilder, R. J., Neidhart, J. A., et al. (1997) IDEC-C2B8 (rituximab) anti-CD20 monoclonal antibody therapy in patients with relapsed low-grade non-Hodgkin's lymphoma. Blood 90 2188-2195.

Maloney, D. G., Liles, T. M., Czerwinski, D. K., Waldichuk, C., Rosenberg, J., Grillo-Lopez, A., et al. (1994). Phase I clinical trial using escalating singledose infusion of chimeric anti-CD20 monoclonal antibody (IDEC-C2B8) in patients with recurrent B-cell lymphoma. Blood 84, 2457-2466.
Marcus, R., Imrie, K., Solal-Celigny, P., Catalano, J. V., Dmoszynska, A., Raposo, J. C., etal. (2008). Phase III study of R-CVP compared with cyclophosphamide, vincristine, and prednisone alone in patients with previously untreated advanced follicular lymphoma. J. Clin. Oncol. 26, 4579-4586.

McLaughlin, P., Grillo-Lopez, A., Link, B., Levy, R., Czuczman, M., Williams, M., et al. (1998). Rituximab chimeric anti-CD20 monoclonal antibody therapy for relapsed indolent lymphoma: half of patients respond to a four-dose treatment program. J. Clin. Oncol. 16, 2825 2833.

O’Brien, M., Easterbrook, P., Powell, J. Blackledge, G., Jones, L., Maclennan, I., et al. (1991). The natural history of low grade non-Hodgkin's lymphoma and the impact of a no initial treatment policy on survival. Q. J. Med. 80, 651-660.

Ogura, M., Tobinai, K., Hatake, K. Uchida, T., Kasai, M., Oyama, T., et al. (2010). Phase I study of inotuzumab ozogamicin (CMC-544) in Japanese patients with follicular lym phoma pretreated with rituximabbased therapy. Cancer Sci. 101, 1840-1845.

Press, O. W., Unger, J. M., Braziel, R. M., Maloney, D. G., Miller, T. P., LeBlanc, M., et al. (2006). Phase II trial of CHOP chemotherapy followed by tositumomab/iodine I-131 tositumomab for previously untreated follicular non-Hodgkin's lymphoma: five-year follow-up of Southwest Oncology Group Protocol S9911. J. Clin. Oncol. 24, 4143-4149.

Press, O. W., Unger, J. M., Rimsza, L. M., Friedberg, J., LeBlanc, M., Czuczman, M. S., et al. (2011). A phase III randomized intergroup trial (SWOG S0016) of CHOP chemotherapy plus rituximab vs. $\mathrm{CHOP}$ chemotherapy plus iodine-131-tositumomab for the treatment of newly diagnosed follicular non-Hodgkin's lymphoma. Blood 118, abstr. 98 .

Price, C. G., Rohatiner, A. Z., Steward, W., Deakin, D., Bailey, N., Norton, A., et al. (1991). Interferon- $\alpha 2 b$ in the treatment of follicular lymphoma: preliminary results of a trial in progress. Ann. Oncol. 2(Suppl. 2), 141-145.

Roulland, S., Navarro, J.-M., Grenot, P., Milili, M., Agopian, J., Montpellier, B., et al. (2006). Follicular lymphoma-like B cells in healthy individuals: a novel intermediate step in early lymphomagenesis. J. Exp. Med. 203, 2425-2431.
Rummel, M. J., Al-Batran, S. E., Kim S-Z, Welslau, M., Hecker, R., Kofahl-Krause, D., et al. (2005). Bendamustine plus rituximab is effective and has a favorable toxicity profile in the treatment of mantle cell and low-grade non-Hodgkin's lymphoma. J. Clin. Oncol. 23, 33833389.

Rummel, M. J., Niederle, N., Maschmeyer, G., Banat, A. G., von Gruenhagen, U., Losem, C., etal. (2012). Bendamustine plus rituximab (B-R) versus $\mathrm{CHOP}$ plus rituximab (CHOP-R) as first-line treatment in patients with indolent and mantle cell lymphomas (MCL): updated results from the StiL NHL1 study. J. Clin. Oncol. 30(Suppl.), abstr. 3 .

Salles, G., Seymour, J. F., Offner, F., Lûpez-Guillermo, A., Belada, D., Xerri, L., etal. (2011). Rituximab maintenance for 2 years in patients with high tumour burden follicular lymphoma responding to rituximab plus chemotherapy (PRIMA): a phase 3 , randomised controlled trial. Lancet 377, 42-51.

Schuster, S. J., Neelapu, S. S., Gause, B. L., Janik, J. E., Muggia, F. M., Gockerman, J. P., et al. (2011). Vaccination with patient-specific tumor-derived antigen in first remission improves disease-free survival in follicular lymphoma. J. Clin. Oncol. 29, 27872794.

Seymour, J. F., Pro, B., Fuller, L. M., Manning, J. T., Hagemeister, F. B., Romaguera, J., et al. (2003). Longterm follow-up of a prospective study of combined modality therapy for stage I-II indolent non-Hodgkin's lymphoma. J. Clin. Oncol. 21, 21152122.

Siegel, R., Ward, E., Brawley, O., and Jemal, A. (2011). Cancer statistics. CA Cancer J. Clin. 61, 212-236.

Solal-Celigny, P., Lepage, E., Brousse, N., Reyes, F., Haioun, C., Leporrier, M., et al. (1993). Recombinant interferon alfa- $2 b$ combined with a regimen containing doxorubicin in patients with advanced follicular lymphoma. N. Engl. J. Med. 329, 16081614.

Solal-Céligny, P., Lepage, E., Brousse, N., Tendler, C. L., Brice, P., Haïoun, C., et al. (1998). Doxorubicincontaining regimen with or without interferon alfa-2b for advanced follicular lymphomas: final analysis of survival and toxicity in the Groupe d'Etude des Lymphomes Folliculaires 86 trial. J. Clin. Oncol. 16, 2332-2338.

Solal-Céligny, P., Roy, P., Colombat, P., White, J., Armitage, J. O., 
Arranz-Saez, R., et al. (2004). Follicular lymphoma international prognostic index. Blood 104, 1258-1265.

Staudt, L. M. (2007). A closer look at follicular lymphoma. N. Engl. J. Med. 356, 741-2.

Staudt, L. M., and Dave, S. (2005). The biology of human lymphoid malignancies revealed by gene expression profiling. Adv. Immunol. 87, 163-208.

Thotathil, Z., and Jameson, M. B. (2007). Early experience with novel immunomodulators for cancer treatment. Expert Opin. Investig. Drugs 16, 1391-1403.

Viardot, A., Goebeler, M., Scheele, J. S., Zugmaier, G., Noppeney, R., Knop, S., et al. (2010). Treatment of patients with non-Hodgkin lymphoma (NHL) with CD19/CD3 bispecific antibody blinatumomab (MT103): double-step dose increase to continuous infusion of $60 \mu \mathrm{m}^{2} / \mathrm{d}$ is tolerable and highly effective. Blood 116, abstr. 2880.

Westin, J. R., Chu, F., Foglietta, M., Rotem-Yehudar, R., and Neelapu, S. S. (2010). Phase II safety and efficacy study of CT-011, a humanized
anti-PD-1 monoclonal antibody, in combination with rituximab in patients with relapsed follicular lymphoma. J. Clin. Oncol. 28(Suppl.), abstr. TPS305.

Wilder, R. B., Jones, D., Tucker, S. L., Fuller, L. M., Ha, C. S., McLaughlin, P., etal. (2001). Long-term results with radiotherapy for stage I-II follicular lymphomas. Int. J. Radiat. Oncol. Biol. Phys. 51, 1219 1227.

Wilson, W., O'Connor, O., Czuczman, M., LaCasce, A., Gerecitano, J., Leonard, J., et al. (2009). Phase $1 / 2$ a study of ABT-263 in relapsed or refractory lymphoid malignancies. Blood 114, abstr. 1711.

Witzig, T. E., Vukov, A. M., Habermann, T. M., Geyer, S., Kurtin, P. J., Friedenberg, W. R., et al. (2005). Rituximab therapy for patients with newly diagnosed, advanced-stage, follicular grade I non-Hodgkin's lymphoma: a phase II trial in the North Central Cancer Treatment Group. J. Clin. Oncol. 23, 1103-1108.

Witzig, T. E., Wiernik, P. H., Moore, T., Reeder, C., Cole, C., Justice, G., et al. (2009). Lenalidomide oral monotherapy produces durable responses in relapsed or refractory indolent non-Hodgkin's lymphoma. J. Clin. Oncol. 27, 54045409.

Wong, E., and Dickinson, M. (2012). Transformation in follicular lymphoma: biology, prognosis, and therapeutic options. Curr. Oncol. Rep. 14 424-432.

Younes, A., Gordon, L., Kim, S. Romaguera, J., Copeland, A. R. de Castro Farial, S., etal. (2009). Phase I multi-dose escalation study of the anti-CD19 maytansinoid immunoconjugate SAR3419 administered by intravenous (IV) infusion every 3 weeks to patients with relapsed/refractory B-cell nonHodgkin's lymphoma (NHL). Blood 114, abstr. 585.

Yuen, A. R., Kamel, O. W., Halpern, J., and Horning, S. J. (1995). Long-term survival after histologic transformation of low-grade follicular lymphoma. J. Clin. Oncol. 13, 17261733.

Zhu, Y. X., Braggio, E., Shi, C.-X., Bruins, L. A., Schmidt, J. E., Van Wier, S., et al. (2011). Cereblon expression is required for the antimyeloma activity of lenalidomide and pomalidomide. Blood 118, 4771-4779.

Conflict of Interest Statement: The authors declare that the research was conducted in the absence of any commercial or financial relationships that could be construed as a potential conflict of interest.

Received: 29 August 2012; accepted: 19 November 2012; published online: 11 December 2012.

Citation: Westin JR and Neelapu SS (2012) Therapy of newly diagnosed follicular lymphoma. Front. Oncol. 2:188. doi: 10.3389/fonc.2012.00188

This article was submitted to Frontiers in Hematology Oncology, a specialty of Frontiers in Oncology.

Copyright (C) 2012 Westin and Neelapu. This is an open-access article distributed under the terms of the Creative Commons Attribution License, which permits use, distribution and reproduction in other forums, provided the original authors and source are credited and subject to any copyright notices concerning any thirdparty graphics etc. 